\title{
Double riches: asteroseismology in eclipsing binaries
}

\author{
John Southworth \\ Astrophysics Group, Keele University, Staffordshire, ST5 5BG, UK \\ email: astro.js@keele.ac.uk
}

\begin{abstract}
The study of eclipsing binaries is our primary source of measured properties of normal stars, achieved through analysis of light and radial velocity curves of eclipsing systems. The study of oscillations and pulsations is increasingly vital for determining the properties of single stars, and investigating the physical phenomena active in their interiors. Combining the two methods holds the promise of establishing stringent tests of stellar evolutionary theory, and of calibrating model-dependent asteroseismology with empirically measured stellar properties. I review recent advances and outline future work.
\end{abstract}

Keywords. stars: binaries: eclipsing, stars: oscillations, stars: fundamental parameters

\section{Introduction}

The study of eclipsing binary star systems (hereafter EBs) has a long history. The eclipse hypothesis was proposed as an explanation of the periodic dimmings of the 'demon star' Algol ( $\beta$ Persei) by John Goodricke (1783). The first empirical measurement of the masses and radii of two stars in an EB was that by Stebbins (1911) for $\beta$ Aurigae; the numbers are close to modern values (Southworth et al. 2007). Shortly afterwards, Russell (1912) established a quantitative method for analysing light curves of eclipses.

The era of high-quality space photometry began in 2006, with a light curve of $\psi$ Centauri from the WIRE satellite (Bruntt et al. 2006). This star shows deep total eclipses on a $38.8 \mathrm{~d}$ period (Fig. 1), and prior to the serendipitous observations from WIRE was not known as either an eclipsing or spectroscopic binary despite its brightness $(V=4.05)$. The current era of vast photometric surveys has led to the discovery of thousands of new EBs, with extensive light curves being obtained particularly by surveys for transiting planets (e.g. TrES, HAT, WASP, CoRoT, Kepler, and in the future TESS and PLATO). Fig. 2 compares the light curves of a transiting planetary system and EB: it is clear that any decent photometric survey for transiting planets is easily capable of detecting eclipsing binaries.

The importance of EBs lies in their amenability to detailed analysis. From fitting a light curve with a simple geometrical model one can determine the fractional radii of the two stars $\left(r_{\mathrm{A}}=\frac{R_{\mathrm{A}}}{a}\right.$ and $r_{\mathrm{B}}=\frac{R_{\mathrm{B}}}{a}$ where $R_{\mathrm{A}, \mathrm{B}}$ are the true radii of the stars and $i$ is the orbital inclination of the system). Spectroscopic radial velocity measurements for both stars allow the minimum masses $\left(M_{\mathrm{A}} \sin ^{3} i\right.$ and $\left.M_{\mathrm{B}} \sin ^{3} i\right)$ and orbital separation $(a \sin i)$ to be calculated. These quantities immediately yield measurements of the masses and radii of the two stars, which can be achieved empirically and to accuracies better than $1 \%$ if the observational data are of good quality (e.g. Clausen et al. 2008).

Obtaining the $T_{\text {eff }}$ values of the stars - procedures for which usually incur some dependence on theoretical models - leads immediately to determination of their luminosities using the formula $L=4 \pi R^{2} T_{\text {eff }}^{4}$, making EBs excellent distance indicators. EB-based distances are available for open clusters as well as for nearby galaxies (e.g. Bonanos et al. 


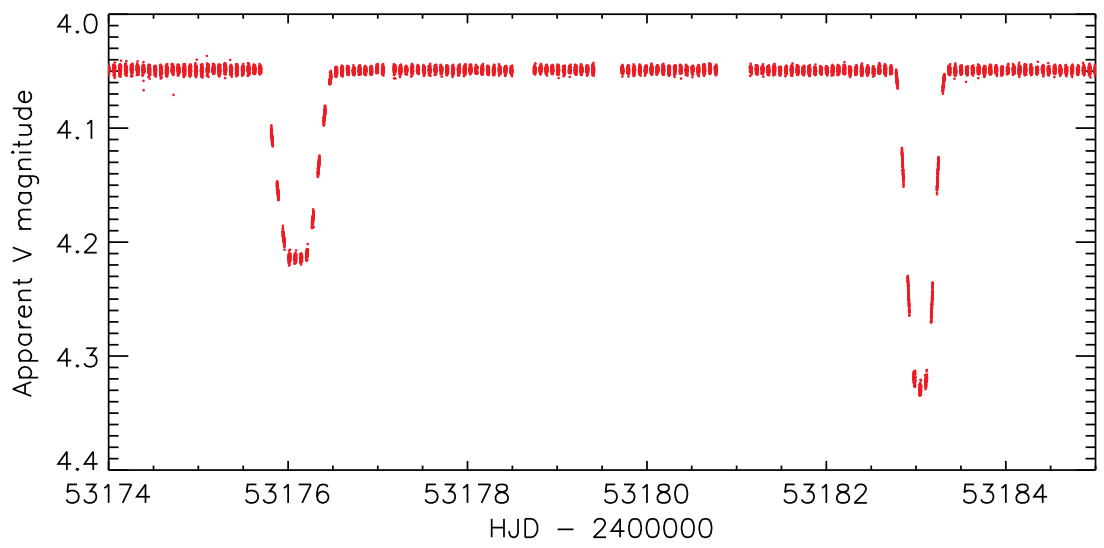

Figure 1. WIRE satellite light curve of the EB $\psi$ Centauri (Bruntt et al. 2006).

2006; North et al. 2010). Another use of EBs is as tests and calibrators of stellar evolutionary theory. The predictions of theoretical models are required to match the measured values of mass, radius, $T_{\text {eff }}$ and luminosity for two stars of the same age and chemical composition (e.g. Pols et al. 1997; Claret 2007).

Many EBs contain stars which show intrinsic variability due to pulsations. Stochastic oscillations are observed in solar-type dwarfs, are present with much higher amplitudes in cool giant stars, and can be used to determine the density, radius and thus mass of the star to high precision (Chaplin et al. 2014). Although of particular importance for solar-type dwarfs, stochastic oscillations have so far only been detected in the giant components of EBs. Other types of pulsations which have been found in conjunction with eclipses are $\delta$ Scuti, $\gamma$ Doradus, spB and tidally-induced oscillations. In this work I review recent results for these objects, then consider the future impact of current and forthcoming space missions.

\section{Red giants in eclipsing binaries}

The one type of system where properties from asteroseismology can currently be compared to those determined via the effects of binarity is that of eclipsing red giants. A single eclipse, $0.12 \mathrm{mag}$ deep and lasting $2.2 \mathrm{~d}$, was found in the Quarter 1 light curve of KIC 8410637 (Hekker et al. 2010) obtained by the Kepler satellite. Subsequent spectroscopic follow-up observations coupled with further eclipses found in the Kepler data have yielded accurate mass and radius measurements for the giant $\left(M_{\mathrm{A}}=\right.$ $\left.1.557 \pm 0.028 \mathrm{M}_{\odot}, R_{\mathrm{A}}=10.74 \pm 0.11 \mathrm{R}_{\odot}\right)$ and its companion $\left(M_{\mathrm{B}}=1.322 \pm 0.017 \mathrm{M}_{\odot}\right.$ and $\left.R_{\mathrm{B}}=1.571 \pm 0.031 \mathrm{R}_{\odot}\right)$, which pursue orbits of period $408.3 \mathrm{~d}$ and eccentricity $e=0.6864 \pm 0.0019$ (Frandsen et al. 2013). The properties of the giant from asteroseismology $\left(1.83 \pm 0.14 \mathrm{M}_{\odot}, 11.58 \pm 0.30 \mathrm{R}_{\odot} ;\right.$ Huber 2015$)$ are larger than the results from the EB analysis by $1.9 \sigma$ and $2.7 \sigma$, respectively, for reasons which are currently under investigation.

Gaulme et al. (2013) has found 12 more similar systems using Kepler data, with orbital periods ranging from $14.7 \mathrm{~d}$ to $1058 \mathrm{~d}$. Gaulme et al. (2014) noticed that the giants in the shorter-period EBs do not show stochastic oscillations, to a detection limit far below the oscillation amplitudes expected for such objects. This suggests that tidal effects spin up the giant, causing magnetic fields which dissipate the acoustic modes. Gaulme et al. (2014) found that mode depletion occurred when the fractional radius of the giant was larger than $0.16-0.24$. 



Figure 2. Kepler satellite light curves of the transiting planetary system Kepler-6 (left) and the EB KIC 5288543 (right) on the same axis scales.

HD 181068 (KIC 5952403) is a bright giant observed by Kepler. It is the primary component of a hierarchical triple system and is orbited by a short-period binary containing two K-dwarfs (Derekas et al. 2011). The two dwarfs are eclipsing on a $0.9 \mathrm{~d}$ period, and themselves transit and are occulted by the G-type giant on a $45.5 \mathrm{~d}$ period. The giant star shows no stochastic oscillations such as are found in single stars of similar mass and radius, but instead shows oscillations at multiples of the orbital frequency. Fuller et al. (2013) found four frequencies in the Kepler Quarter 1-11 data: all are related to the orbital frequency and at least two are tidal in origin. Borkovits et al. (2013) determined the full physical properties of this triple system by using eclipse timing variations in the short-period EB as a substitute for RVs of the two dwarfs, which are unavailable due to their relative faintness. Tidally-induced pulsations have previously been seen in HD 174884 (CoRoT 7758), an EB consisting of two unevolved B-stars (Maceroni et al. 2009).

\section{3. $\delta$ Scuti stars in eclipsing binaries}

$\delta$ Scuti stars are A- and F-type dwarfs which pulsate in non-radial $p$-modes with periods from $0.014 \mathrm{~d}$ to $0.333 \mathrm{~d}$ (Grigahcène et al. 2010). A substantial number of known $\delta$ Scuti stars are members of EBs, including both detached and semi-detached systems. Mkrtichian et al. (2002) defined the class of $\delta$ Scuti stars in semi-detached systems, which have since been labelled as 'oEA' (oscillating Algol) stars. Recent advances in the asteroseismic analysis of $\delta$ Scuti stars have enabled the detection of large frequency separations analogous to solar-like oscillations but in a different pulsation regime (García Hernández et al. 2013), representing a step towards utilising these pulsators as tests of stellar physics. García Hernández et al. (2015) have used EBs observed with Kepler and CoRoT to define a relation between the large frequency separation and mean density for $\delta$ Scuti stars.

An early result from the Kepler satellite was the detection of eclipses and 68 pulsation frequencies in the light curve of KIC 10661783 (Southworth et al. 2011). Follow-up spectroscopy by Lehmann et al. (2013) allowed determination of the masses and radii of the two stars. The secondary component appears to be a classic example of close-binary evolution, having a mass of $0.1913 \pm 0.0025 \mathrm{M}_{\odot}$ and a radius of $1.124 \pm 0.019 \mathrm{R}_{\odot}$. However, the spectroscopic mass ratio leads to a light curve model in which the two stars are currently detached and therefore not undergoing mass transfer. KIC 10661783 may be an example of a semi-detached system which shows only episodic mass transfer. 

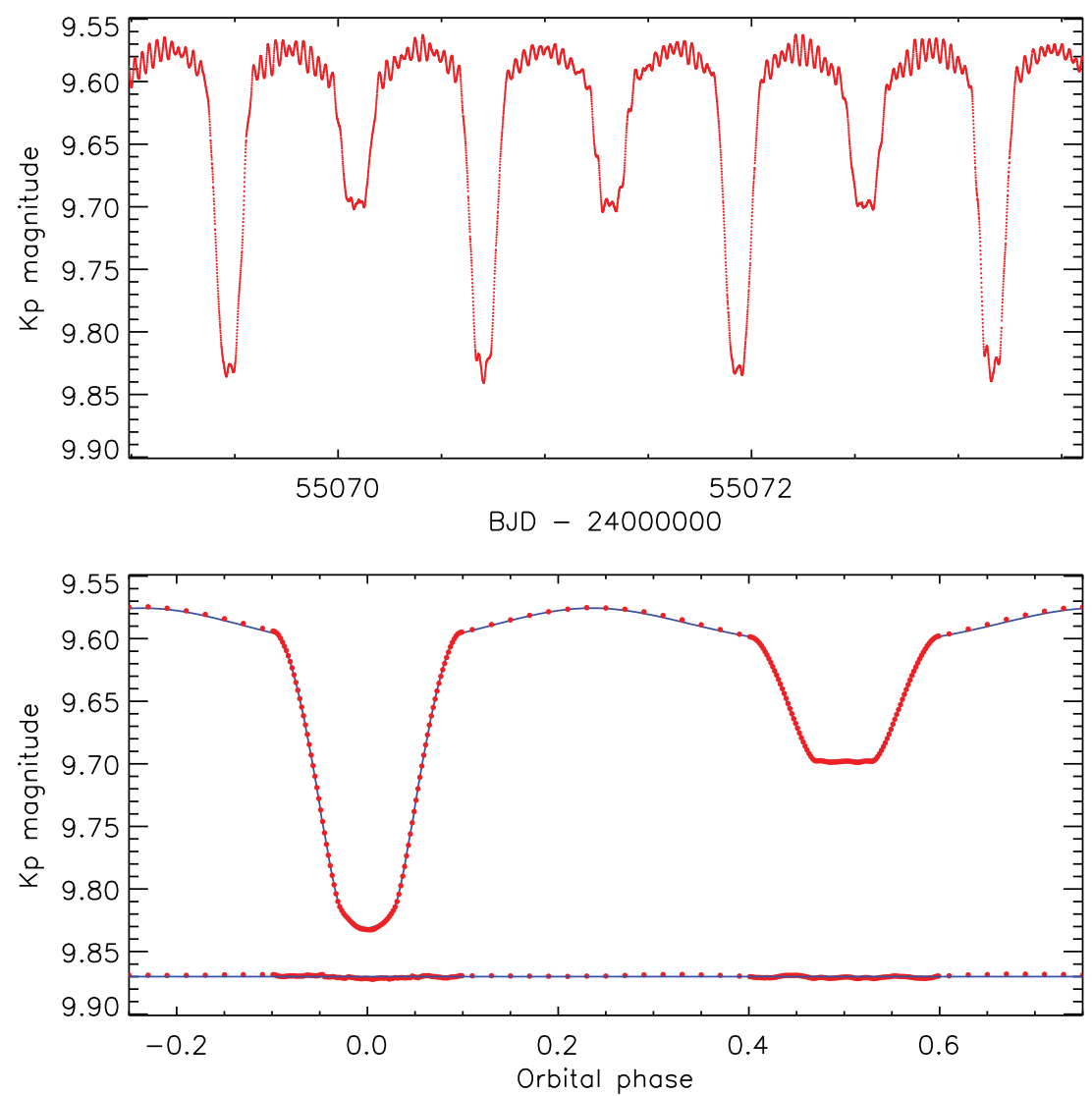

Figure 3. Kepler light curve of the $\delta$ Scuti EB KIC 10661783 (Southworth et al. 2011). Top: part of the short-cadence observations. Bottom: phased light curve after removal of the pulsations.

Other examples include KIC 3858884 (Maceroni et al. 2014), a detached EB with an orbital period of $26.0 \mathrm{~d}$ and a high eccentricity $(e=0.47)$. Theoretical models require convective core overshooting to match the properties of this system. CoRoT 105906206 is another, for which da Silva et al. (2014) required the inclusion of Doppler beaming to fit the light curve.

\section{4. $\gamma$ Doradus stars in eclipsing binaries}

$\gamma$ Dor stars are non-radial $g$-mode pulsators with typical periods of $0.4 \mathrm{~d}$ to $3 \mathrm{~d}$ (Handler 1999) and spectral types of A7-F5 V-IV. The advent of high-precision photometry from space satellites has revealed that a large fraction are hybrids which show both $\gamma$ Dor and $\delta$ Scuti pulsations (Grigahcène et al. 2010). Recent advances in asteroseismology have enabled the detection of period spacings which hold the prospect of detailed seismic analyses of these objects (Van Reeth et al. 2015; Kurtz et al. 2015).

It was inevitable that some $\gamma$ Dor stars would be found in EBs, and cases have indeed been identified from Kepler observations. Debosscher et al. (2013) presented a detailed analysis of KIC 11285625 , which shows $12 \%$-deep eclipses on a $10.8 \mathrm{~d}$ period plus $g$-mode oscillations of similar amplitude (Fig. 4). Analysis of the Kepler data and ground-based radial velocity measurements allowed Debosscher et al. to determine the masses and 


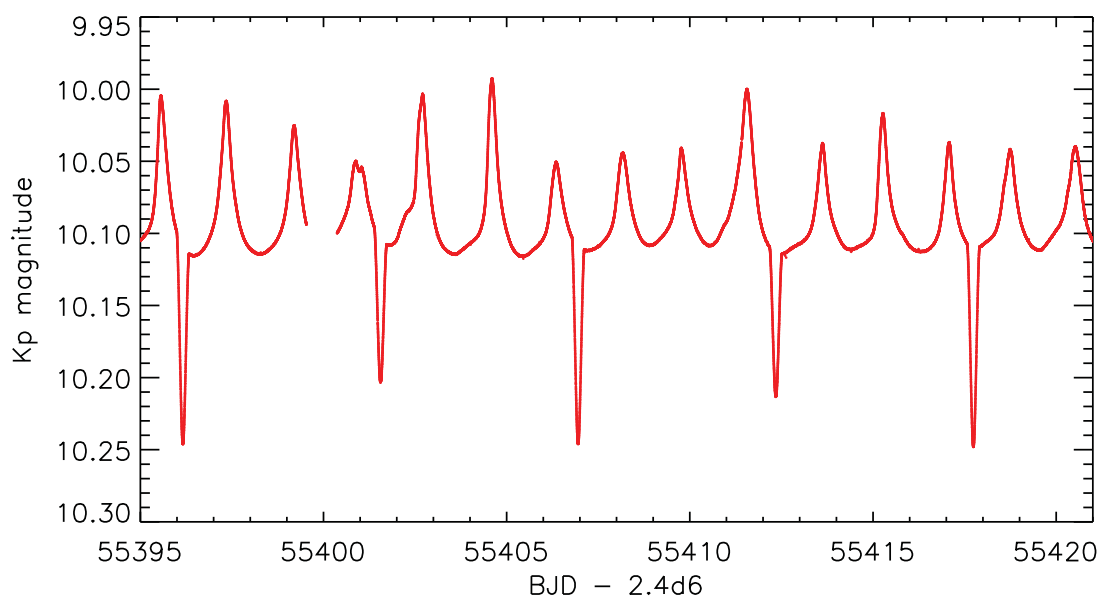

Figure 4. Kepler light curve of KIC 11285625, which shows eclipses and $\gamma$ Dor pulsations.
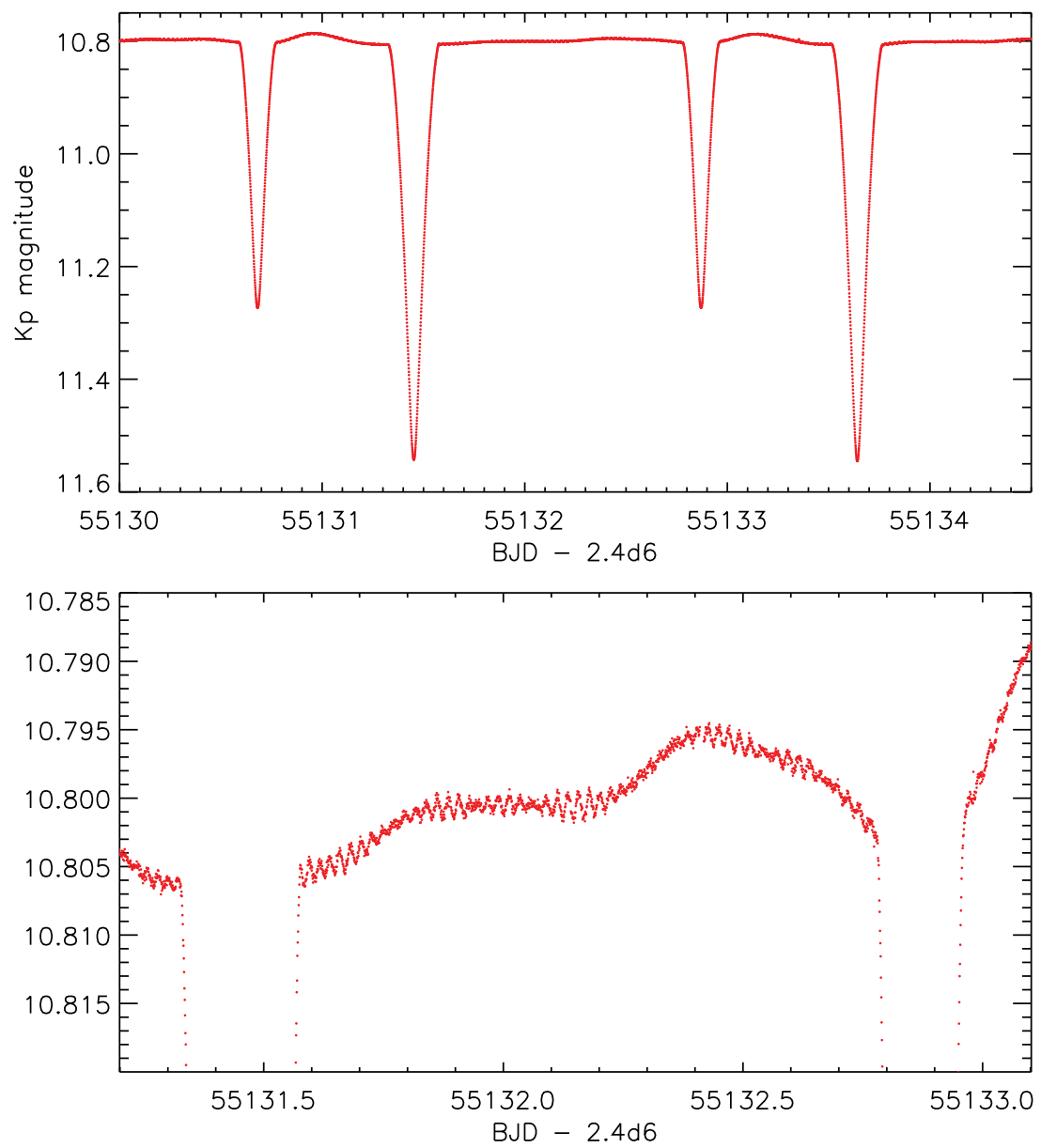

Figure 5. Kepler light curve of KIC 4544587 , which shows eclipses, $\delta$ Scuti and $\gamma$ Dor pulsations. Top:part of the short-cadence observations. Bottom: close-up of an short stretch of data outside eclipse. 


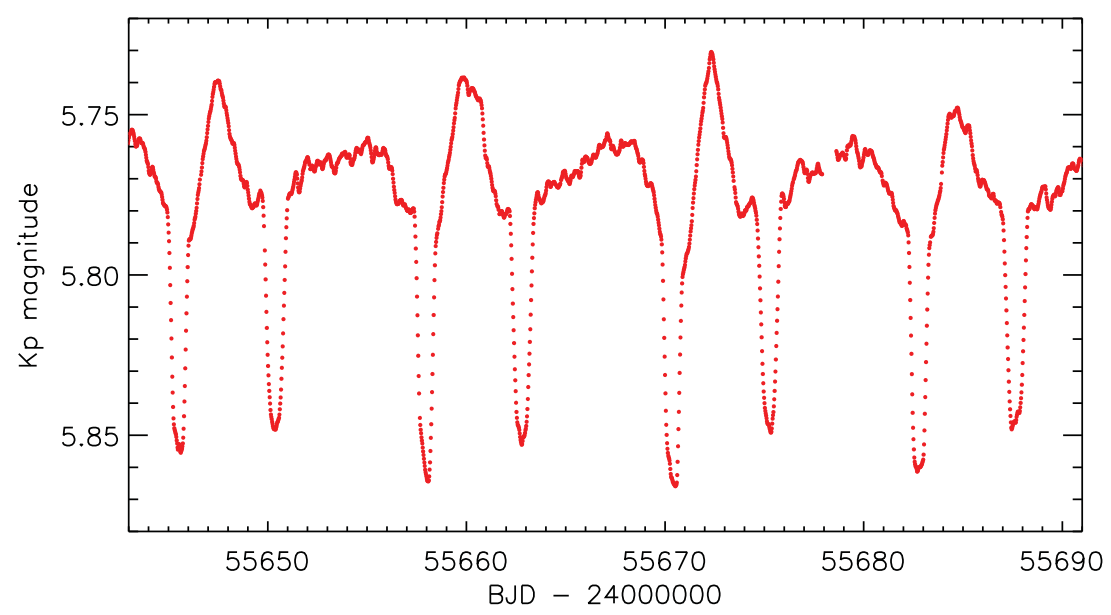

Figure 6. Fragment of the Kepler short-cadence light curve of V380 Cygni.

radii of the two component of the $\mathrm{EB}$ to $1 \%$. Amplitude modulation and splitting at the rotational and orbital frequencies was also found from analysis of the pulsations.

Another example is KIC 4544587 (Hambleton et al. 2013), an EB with an eccentric $2.2 \mathrm{~d}$ period orbit and rapid apsidal motion. Whilst the eclipses dominate the Kepler light curve (Fig. 5), both $\delta$ Scuti and $\gamma$ Dor pulsations are visible on closer inspection. (Hambleton et al. 2013) found $14 \mathrm{~g}$-modes, most of which were harmonics of the orbital frequency, and $17 p$-modes which were either affected by tides or occurring within the secondary star.

\section{Stochastic oscillations in eclipsing binaries}

A major goal for space-based transit surveys such as Kepler and PLATO is the identification of potentially habitable planets. The widely accepted criteria for such planets is that they are Earth-like: rocky and orbiting within the habitable zone of their host star (e.g. Rauer et al. 2014). The low masses and radii of such planets, coupled with their long orbital periods, makes them difficult to characterise. Their radii will be measured relative to those of their host stars, so it is important to obtain as much information as possible about these stars. Asteroseismology is an important component of such work (e.g. Christensen-Dalsgaard et al. 2010; Borucki et al. 2012), and EBs offer an unrivalled opportunity to check the reliability of the asteroseismic results.

However, this has yet to be achieved because stochastic oscillations have not been detected in a solar-type eclipsing binary. The short-period EBs observed using Kepler suffer from tidal effects which cause rotational splitting and dissipation of the acoustic modes, making them undetectable in current data. The scarcity of longer-period EBs (orbital period greater than approximately $10 \mathrm{~d}$ depending on the masses and evolutionary statuses of the components) means that no suitable candidates have been found for a comparison between asteroseismic properties and those measured from eclipses and radial velocities.

One notable success has been the detection of stochastic oscillations in the extremely bright $(V=5.7)$ totally-eclipsing binary V380 Cygni (Tkachenko et al. 2012, 2014), which is composed of a B1.5 giant and a B2 dwarf of known chemical composition (Pavlovski et al. 2009). Fig. 6 shows part of the Kepler light curve of this system, in which can be seen primary and secondary eclipses, periastron brightening due to tidal effects, and stochastic variability. A Fourier transform of the Kepler light curve after subtraction 
of the effects of binarity shows some frequencies which are multiples of the orbital frequency and others which are not. The physical properties of the binary can only be matched by theoretical models with unrealistically high rotation and strong convective core overshooting, implying that much increased mixing is needed in these models.

\section{Present and future}

Although this review has showcased many results based on data from the Kepler satellite, the archive of Kepler data contains over 2700 EBs (Kirk et al., in press) of which the vast majority have not received significant attention. The CoRoT satellite also obtained over 163000 light curves including over 3000 EBs (Moutou et al. 2013), and the $\mathrm{K} 2$ mission is continuing to accumulate copious high-quality space-based data (Howell et al. 2014; Conroy et al. 2014). The near future holds the prospect of the TESS satellite, which will observe most of the sky for $27 \mathrm{~d}$ intervals and thus greatly expand the database for bright EBs.

One of the remaining tasks is to study solar-type EBs for which stochastic oscillations can be detected. This necessarily requires space-based photometry in order to get observations with a high duty cycle, low scatter, and long time coverage. Each of the past and present space missions has at least one feature which compromises its ability to perform this experiment, such as low sampling rate (most of the Kepler targets), faintness of the stars observed (Kepler and CoRoT), or insufficient duration of observations (TESS, except for small patches of sky near the celestial poles).

The PLATO mission promises to solve all of these problems (Rauer et al. 2014). Scheduled for launch in 2024, it will observe hundreds of thousands of bright stars at high cadence and for time intervals up to several years. Extensive effort will be needed to cope with this inundation of data, and to procure follow-up observations from ground-based facilities. The work package structure for PLATO includes one dedicated to binary and multiple stars, for which the author is responsible, within the Complementary Science Work Package group. Although preparation of an extensive target list of known EBs will form part of this work package, it is likely that the majority of EBs observed by PLATO will be previously unidentified binaries selected for observation in one of the main transit search samples. These will, by design, be F, G, K and M dwarfs and the study of those showing eclipses promises to revolutionise our understanding of solar-type and low-mass stars.

\section{References}

Bonanos, A. Z., et al., 2006, ApJ, 652, 313

Borkovits, T., et al., 2013, MNRAS, 428, 1656

Borucki, W. J., et al., 2012, ApJ, 745, 120

Bruntt, H., Southworth, J., Torres, G., Penny, A. J., Clausen, J. V., \& Buzasi, D. L., 2006, $A \mathscr{G} A, 456,651$

Chaplin, W. J., et al., 2014, ApJS, 210, 1

Christensen-Dalsgaard, J., et al., 2010, ApJ, 713, L164

Claret, A., 2007, A\&A, 475, 1019

Clausen, J. V., Torres, G., Bruntt, H., Andersen, J., Nordström, B., Stefanik, R. P., Latham, D. W., \& Southworth, J., 2008, A\&A, 487, 1095

Conroy, K. E., et al., 2014, PASP, 126, 914

da Silva, R., Maceroni, C., Gandolfi, D., Lehmann, H., \& Hatzes, A. P., 2014, A\& A, 565, A55

Debosscher, J., et al., 2013, A\&A, 556, A56

Derekas, A., et al., 2011, Science, 332, 216 
Frandsen, S., et al., 2013, A\&̈A, 556, A138

Fuller, J., Derekas, A., Borkovits, T., Huber, D., Bedding, T. R., \& Kiss, L. L., 2013, MNRAS, 429,2425

García Hernández, A., Martín-Ruiz, S., Monteiro, M. J. P. F. G., Suárez, J. C., Reese, D. R., Pascual-Granado, J., \& Garrido, R., 2015, ApJL, in press, arXiv:1509.01111

García Hernández, A., et al., 2013, A\&̈A, 559, A63

Gaulme, P., McKeever, J., Rawls, M. L., Jackiewicz, J., Mosser, B., \& Guzik, J. A., 2013, ApJ, 767,82

Gaulme, P., Jackiewicz, J., Appourchaux, T., \& Mosser, B., 2014, ApJ, 785, 5

Goodricke, J., 1783, Royal Society of London Philosophical Transactions Series I, 73, 474

Grigahcène, A., et al., 2010, ApJ, 713, L192

Hambleton, K. M., et al., 2013, MNRAS, 434, 925

Handler, G., 1999, MNRAS, 309, L19

Hekker, S., et al., 2010, ApJ, 713, L187

Howell, S. B., et al., 2014, PASP, 126, 398

Huber, D., 2015, in Astrophysics and Space Science Library, vol. 408 of Astrophysics and Space Science Library, p. 169

Kurtz, D. W., Shibahashi, H., Murphy, S. J., Bedding, T. R., \& Bowman, D. M., 2015, MNRAS, 450,3015

Lehmann, H., Southworth, J., Tkachenko, A., \& Pavlovski, K., 2013, A\&A, 557, A79

Maceroni, C., et al., 2009, A\&A, 508, 1375

Maceroni, C., et al., 2014, A\&SA, 563, A59

Mkrtichian, D. E., Kusakin, A. V., Gamarova, A. Y., \& Nazarenko, V., 2002, in C. Aerts, T. R. Bedding, \& J. Christensen-Dalsgaard, ed., IAU Colloq. 185: Radial and Nonradial Pulsations as Probes of Stellar Physics, vol. 259 of Astronomical Society of the Pacific Conference Series, p. 96

Moutou, C., et al., 2013, Icarus, 226, 1625

North, P., Gauderon, R., Barblan, F., \& Royer, F., 2010, A\&A A, 520, A74

Pavlovski, K., Tamajo, E., Koubský, P., Southworth, J., Yang, S., \& Kolbas, V., 2009, MNRAS, 400, 791

Pols, O. R., Tout, C. A., Schroder, K.-P., Eggleton, P. P., \& Manners, J., 1997, MNRAS, 289, 869

Rauer, H., et al., 2014, Experimental Astronomy, 38, 249

Russell, H. N., 1912, ApJ, 35, 315

Southworth, J., Bruntt, H., \& Buzasi, D. L., 2007, A\&A A, 467, 1215

Southworth, J., et al., 2011, MNRAS, 414, 2413

Stebbins, J., 1911, ApJ, 34, 112

Tkachenko, A., et al., 2012, MNRAS, 424, L21

Tkachenko, A., et al., 2014, MNRAS, 438, 3093

Van Reeth, T., et al., 2015, A\&SA, 574, A17 